\title{
The Learning Experience With Electronic Museum Guides
}

\author{
Christoph Bartneck \\ Eindhoven University of Technology
}

Aya Masuoka, Toru Takahashi, and Takugo Fukaya

Advanced Telecommunication Institute International

\begin{abstract}
Within the contextual model of learning framework, the authors conducted a study with electronic handheld guides at the Van Abbemuseum (Eindhoven, the Netherlands). This study investigated the influence that limiting users' freedom of choice and control by facilitated mediation of others has on users' learning experiences. The different levels of control were implemented on the ubiNext museum guide system. The level of users' control had no significant influence on the amount of art the participants talked about. However, in the curator recommendation mode, the participants had a more passive attitude. The different handheld devices did not perform better than the paper guide. As the paper guide did not require any troubleshooting, it can even be considered a better solution. However, the handheld devices could not be used to their full potential as audio and video data were not present.
\end{abstract}

Keywords: museum, electronic, guide, PDA, learning

Museums increasingly acknowledge the need to provide adequate educational services to their visitors. The times of selfsufficient egalitarian arrogance, in which visitors were left alone with undecipherable art objects, are, one hopes, over. To guide museums' educational efforts, first theoretical frameworks became available. The contextual model of learning framework is among the most renowned (Falk \& Dierking, 2000). It distinguishes 11 key factors that influence visitors' learning experiences: Motivation and Expectations (Factor 1), Interest (Factor 2), Prior Knowledge and Experience (Factor 3), Choice and Control (Factor 4), Within-Group Sociocultural Mediation (Factor 5), Facilitated Mediation by Others (Factor 6), Culture (Factor 7), Advance Preparation (Factor 8), Setting (Factor 9), Design (Factor 10), and Subsequent Reinforcing Events and Experiences (Factor 11). Three of these factors are of special interest to this study.

Factor 3 (Prior Knowledge and Experience) deals with the widely known fact (Roschelle, 1995) that people who have prior knowledge and experience with art objects are better able to create meaning from new ones.

Factor 4 (Choice and Control) refers to a situation in which individuals can exercise choice over what, how, when, and with whom they learn and feel in control of their own learning. It has been shown that children prefer to visit museums with their families, compared with school trips, because they have more control over the experience (Griffin, 1996; Griffin, 1998; Jensen, 1994).

Christoph Bartneck, Department of Industrial Design, Eindhoven University of Technology, Eindhoven, the Netherlands; Aya Masuoka, Toru Takahashi, and Takugo Fukaya, Media Information Science Laboratory, Advanced Telecommunication Institute International, Kyoto, Japan.

We would like to thank the following persons (in alphabetical order) for supporting this study: Marion de Groof, John Helmes, Loes Janssen, Willem Jan Renders, and Machi Takahashi. This research was partly supported by the National Institute of Information and Communications Technology (NICT) of Japan.

Correspondence concerning this article should be addressed to Christoph Bartneck, Department of Industrial Design, Eindhoven University of Technology, Den Dolech 2, 5600 MB, Eindhoven, the Netherlands. E-mail: christoph@bartneck.de.
Factor 6 (Facilitated Mediation by Others) describes a situation in which people perceived to be knowledgeable, such as teachers and parents, facilitate the learning experience. This classic scenario has a long cultural history and, if done right, can be very supportive.

These three factors promote visitors' learning experience, but unfortunately Factors 4 and 6 have an antiproportional relationship. The more the visitors are facilitated by, for example, a museum docent, the less choice and control they have. A careful balance between Factors 4 and 6 is necessary to maximize visitors' learning experience. For example, the museum's staff could provide explanations only on request by the visitor.

It has to be acknowledged that the concept of "learning experience" remains on an abstract level. Not just the repeatable knowledge of art, such as the recognition of art objects and their key data, is considered a learning experience, but also personal experiences that might influence the visitors' behavior in the future (Falk \& Dierking, 2000). A visitor might, for example, remember the atmosphere of a Picasso painting years after visiting the exhibition and use this knowledge to interpret another art object or decide to spend a vacation in France.

The operationalization of such an abstract concept is difficult. Still, certain visitor behaviors are fairly good indicators for it. Clearly, the longer visitors indulge in an exhibition to see many art objects, the higher the chance that they had a learning experience (Smith \& Smith, 2003). Furthermore, the more discussions and reflections the visitors have with each other about the exhibited art, the more likely they had a learning experience (Smith \& Smith, 2003). This indicator is explicitly mentioned as Factor 5 (WithinGroup Sociocultural Mediation) in the contextual model of learning framework. In summary, this study investigated the influence that limiting the users' freedom of choice and control (user control) by facilitated mediation by others has on the users' learning experience.

To create different levels of facilitation and thereby different levels of user control, we used the ubiNext museum guide system developed in the Media Information Science laboratories of the Advanced Telecommunication Institute International, Kyoto, Ja- 
pan. We highlight only the most relevant features of the system here, but the interested reader might consult Masuoka, Fukaya, Takahashi, Takahashi, and Ito (2005) for a detailed description. UbiNext is a museum guide system that uses personal digital assistants (PDAs) to provide the user with information about art objects and recommendations on what other objects the user might be interested in.

The system can be operated in different modes that give the user more or less control. The least facilitated mode and therefore the one with the highest user control is the "no recommendation" (noReco) mode. Users may freely walk through the exhibition, and whenever they are interested in an art object they may request information about it on the PDA. The second least facilitated mode is the "ubiNext recommendation" mode (ubiNextReco). In this mode, the system recommends art objects to the user on the basis of certain parameters, such as their previously expressed object ratings and their current location. The system allows the user not to follow the recommendation, and the user may still request information about other art objects. The "self-recommendation" mode (selfReco) is slightly more restrictive. Before visiting the museum, users create their personal tour through ubiNext's previsit planning Web site. When entering the exhibition, the system guides the users along their personal tours and does not allow them to deviate from it. If a user requests information about an object that is not in the tour, ubiNext would give the most basic information but would only offer a back button to return to the tour. The most facilitating and thereby most restrictive mode is the "curator recommendation" mode (curatorReco). The curator of the exhibition creates a tour and, similar to the selfReco mode, the users are guided along it. Again, the users are not allowed to deviate from the tour.

Although a PDA-based system is ideal for implementing different levels of facilitation, it does contain some possibly problematic attributes. Users need to learn how to interact with the device, which might be difficult for older visitors. In comparison, paperbased guides, such as booklets or brochures, are certainly easier to use and might make users feel more comfortable. Comfort as described in Factor 9 (Setting) is expected to promote learning experiences; therefore, PDA-based systems might be less suitable. We therefore included a paper guide version in this study.

Another important attribute of PDAs is their screen size. A larger screen might enable users to easily share information and might even encourage them to hold the device together. This increased cooperation may lead to a better learning experience, as described in Factor 5, Within-Group Sociocultural Mediation. We therefore included a standard PDA and the considerably larger ProScribe in this study (see Figure 1 and Figure 2). The ProScribe's screen is approximately the size of A4 paper and is therefore suitable for a comparison to a paper guide.

Obviously, PDAs require a high initial investment and might also cause higher maintenance costs compared with paper-based guides or even audio tours. Although this financial aspect is certainly of relevance to the management of museums, it is not within the scope of this study. A thorough economic assessment that evaluates the total cost of ownership would be necessary, and we hope that such a study becomes available in the future.

ubiNext contains the ubiNextReco mode, in which the system gives recommendations to the user on what art object to look at next. These recommendations are based on an algorithm that does

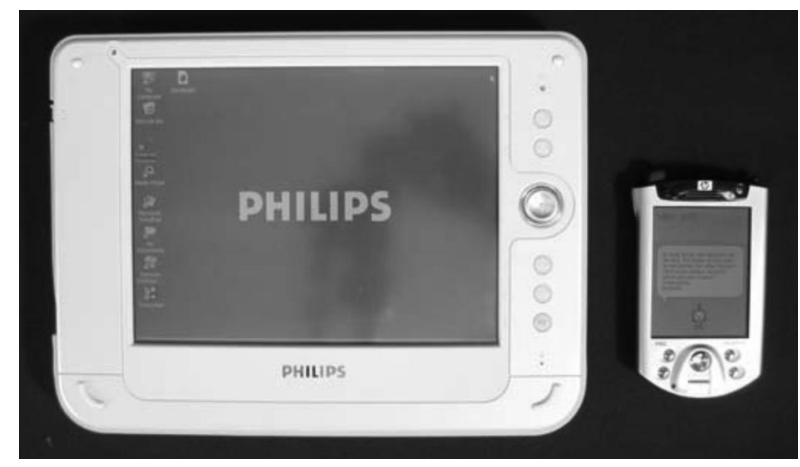

Figure 1. The ProScribe and the personal digital assistant.

consider the previously given user ratings and the spatial distance of the user to the art objects. The advantage of such an algorithmic approach is that personal and context-relevant recommendations can be given. Only a personal human tour guide could otherwise achieve a similar effect. The question remains whether the quality of the algorithmic recommendation is good enough compared with the recommendations given through a regular museum tour.

As described by Factor 3, prior knowledge and experience improves the museum learning experience. We therefore were interested in whether users who worked with ubiNext's previsit planning Web site would have a better learning experience compared with users who did not use the previsit planning Web site.

On the basis of the issues mentioned above, we defined the following research questions for this study:

1. What influence does the level of user control (Choice and Control) have on the user's learning experience?

2. Does a PDA-based solution improve the user's learning experience as compared with a paper-and-pen solution?

3. Does a larger PDA screen improve the user's learning experience?

4. Do ubiNext's algorithmic recommendations lead to a better learning experience as compared with the museum's recommendations?

5. Does ubiNext's previsit tour-planning Web site improve the user's learning experience?

\section{Method}

\section{Conditions}

We conducted a 3 (technology) $\times 4$ (user control) $\times 2$ (preparation) between-participants experiment. Within the user control factor, the following conditions were presented to the participants:

- No recommendation (noReco) - The system would not give any recommendation. The users had to request information on every art object by themselves.

- ubiNext recommendation (ubiNextReco)—The system would give recommendations on what art objects might interest the participants, based on an internal algorithm.

- Self-recommendation (selfReco)_Before their exhibition visit, the 


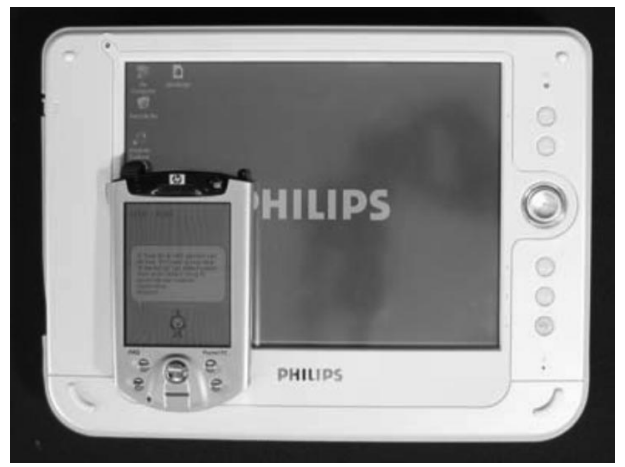

Figure 2. Comparison of the personal digital assistant and the ProScribe.

participants created their own tours. The PDA then recommended the art objects of this tour in a set sequence.

- Curator recommendation (curatorReco)-The education staff of the exhibition created a tour with great care. The system would then recommend the art objects of this tour in a fixed sequence.

The technology conditions consisted of the selfReco condition mentioned above and the following conditions:

- Self-recommendation using ProScribe (selfRecoProScribe)-The system behaved similarly to the selfReco condition, but instead of using a small PDA, the participant used a ProScribe.

- Self-recommendation using paper and pen (selfRecoPaper)—Before visiting the exhibition, the participants planned their own tour using a paper-and-pen guide. They then took their planned paper-based tour to the exhibition.

The preparation conditions were available within the six conditions mentioned so far. All of the technology conditions (selfReco, selfRecoProScribe, and selfRecoPaper) required the users to prepare their tours before the museum visit and therefore formed the preparationSelf condition. The noReco, ubiNextReco, and curatorReco conditions were not prepared by the user. They therefore formed the preparationOther condition. Table 1 gives an overview of the study conditions.

\section{Measurements}

In this section, italics are used to highlight the measurements' names.

Demographics. Age and gender of the participants were recorded. The genders of couples were recorded as male, mixed, or female.

Conversation. The participants' utterances were recorded on a small audio recorder that was hung around the neck of one participant. After the experiment, the utterances were analyzed. First, the recording was cropped from the point when the participants entered the exhibition to when they exited the exhibition. The duration was noted as speechTotal. The utterances in the recording were then grouped into the following categories, and duration of the categories was then noted. Therefore, the sum of how long participants talked about each category was measured, not the number of utterances in a category.

- Art-The participants talked about art objects, related concepts, and their reaction to them.

- Handheld - The participants discussed the functioning of the handheld device.

- Navigation-The participants conversed about where they were and where they wanted to go.

- notAudible - This includes utterances that could not be understood, for example, when the participants spoke too softly.

- offTopic-The participants talked about topics that were not connected to the exhibition of the study. For example, the participants talked about how much or how little they liked a certain teacher.

- Silence-A pause of $2 \mathrm{~s}$ and longer was defined as silence. This is a standard threshold in speech analysis. Pauses shorter than $2 \mathrm{~s}$ are therefore considered to be part of the conversation flow in the other categories.

- Troubleshooting-The participants talked about technical problems and may have asked the experimenter for help.

When two or more groups of participants talked to each other, the utterances were categorized as groupInteraction. Within groupInteraction, the same subcategories exist as used above: groupArt, groupHandheld, groupNavigation, groupNotAudible, groupOffTopic, groupSilence, and groupTroubleshooting.

Behavior. Every time the participants executed an action on the handheld device, such as requesting information about an art object, a log file entry on the server was automatically created. On the basis of this log file data, several measurements were available:

- durationOfVisit - the duration from the first user action to the last user action. This measurement is a subsection of speechTotal.

- numberOfObjects - the number of objects that the participant requested information about

- averageDurationAtObject - the average duration between requests for information about objects. This measurement includes the duration of walking from one object to the next. Given the relatively small exhibition space, the variations in the walking duration may be neglected.

- numberOfQuizzes - the number of quizzes the participants took

- percentageOfQuizzes - the percentage of quizzes taken. This measurement was calculated by dividing the numberOfObjects by the numberOfQuizzes.

- numberOfSelectionQuizzes - the number of selection quizzes within the numberOfQuizzes the participants took. Only quizzes that allowed the user to give an answer were considered selection quizzes.

- numberOfRightAnswers - the number of correct answers given to the selection quizzes

- percentageRightAnswers - the percentage of correct answers given to the selection quizzes

- numberOfRecommendation - the number of recommendation the system made. Recommendations could come from the algorithm (ubiNextReco), from a personal tour (selfReco), or from the museum (curatorReco).

Table 1

The Conditions of the Experiment

\begin{tabular}{|c|c|c|c|c|}
\hline \multirow[b]{2}{*}{ User control } & \multicolumn{3}{|c|}{ Technology } & \multirow[b]{2}{*}{ Preparation } \\
\hline & PDA & ProScribe & Paper & \\
\hline No recommendation & noReco & & & Other \\
\hline ubiNext recommendation & ubiNextReco & & & Other \\
\hline Self-recommendation & selfReco & selfRecoProScribe & selfRecoPaper & Self \\
\hline Curator recommendation & curatorReco & & & Other \\
\hline
\end{tabular}

Note. $\quad \mathrm{PDA}=$ personal digital assistant. 
- numberOfFollowedRecommedation - the number of recommendations the participant followed

These measurements contain a certain number of errors, as a participant could request the same documents several times. Owing to technical problems, a request might also have been reloaded by the system. The size of this error is not clear, but it can be assumed that it is evenly distributed.

These measurements are not available for the selfRecoPaper condition, as the participants in this condition did not use a handheld device. Only the demographic and conversation measurements were available for this condition.

\section{Participants}

One hundred forty-eight (76 female and 72 male) local school children between the ages of 14 and $18(M=14.79$ years, $S D=0.44)$ participated in this study in the framework of their art classes. The students were part of two different school levels. Sixty-eight were part of the more applied "Havo" school, and 80 were part of the more academically oriented "Gymnasium." They did not receive a reward for their participation.

\section{Setup}

This study was executed at the Van Abbemuseum in Eindhoven, the Netherlands. The experiment was confined to the El Lissitzky exhibition within the museum. The exhibition consisted of three rooms containing paintings, sculptures, and installations. Figure 3 shows a few examples of objects in the exhibition.

Owing to the exhibition space's limited size, the participants could move quickly from one art object to another. Figure 4 shows the map of the exhibition. A wireless network access point was installed in each room to guarantee optimal network performance.

Twenty art objects out of the approximately 70 objects in the exhibition were available in the system. Labels containing the identification number of the object were attached below the regular art object labels.

Six regular PDAs (Hewlett-Packard iPAQ Pocket PC h5550) and two ProScribes (Philips 100WT10P) were used in the study (see Figure 1). All of them had built-in wireless network capability through which they communicated with the server.

The PDA's screen was $240 \times 320$ pixels and had a diagonal of approximately $9.9 \mathrm{~cm}$. Therefore, the PDA had a screen resolution of nearly 100 dpi. The ProScribe's screen was $600 \times 800$ pixels and had a diagonal of $26.4 \mathrm{~cm}$. Figure 2 shows the difference in size between the PDA and the ProScribe. The PDA weighed $207 \mathrm{~g}$ and the ProScribe 1,600 g. At this point in time, a comparable PDA costs approximately 400 Euros (approximately \$503), whereas the ProScribe costs around 1,300 Euros (approximately $\$ 1,636)$. Two different designs displayed the content optimally for the two different devices. The text for the ProScribe, for example, was much larger in terms of pixels than that of the PDA.

All buttons of the devices were disabled and the Web browser was set into kiosk mode. The participants could therefore not use any browser button or other application on the device or manipulate its system settings. The participants interacted with the software by clicking on the touch screen using the devices' touch pens (see Figure 5). The interface elements and all displayed content were in the participants' native language.
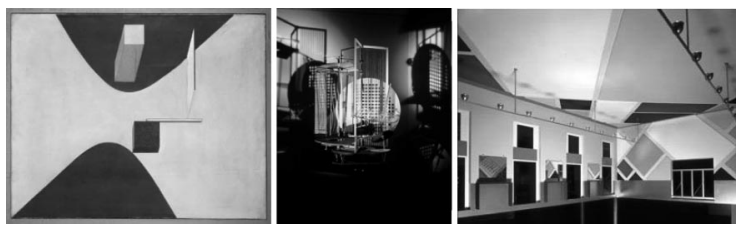

Figure 3. Examples of objects in the exhibition.

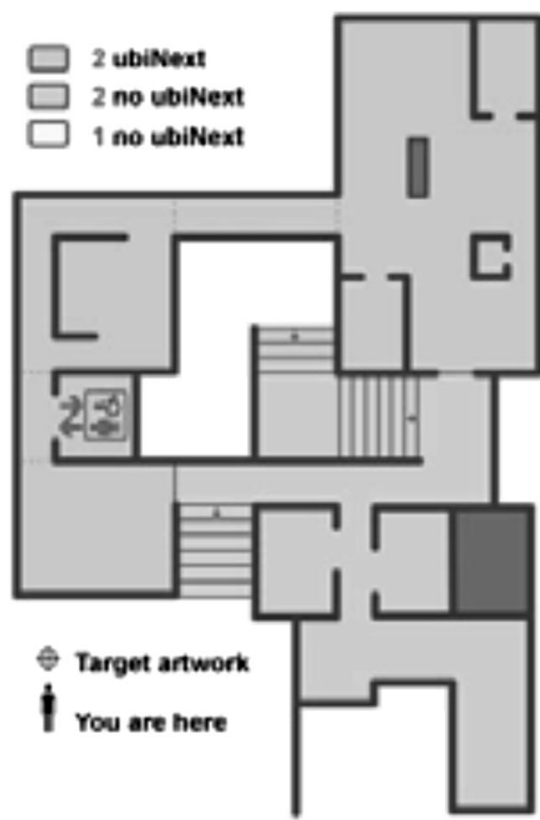

Figure 4. Map of the exhibition space.

The ubiNext recommendation algorithm considers user preference, the spatial distance between the user and the various art objects, and the internal relationship of the art objects, as defined through the assigned keywords into consideration.

Participants' conversations were recorded using a small MP3 player (Creative MuVo V200) that was hung around their necks. The device's built-in microphone provided sufficient audio quality for the recording. The recordings were analyzed after the experiment using Noldus Observer.

\section{Procedure}

Two weeks before the experiment, the art teachers informed the students in six different art classes about the study. For their homework, five classes were asked to visit ubiNext's previsit planning Web site to investigate the various art objects in the exhibition and prepare a tour for themselves. One class was given the paper guide with the same task. If a class had been confronted with both the Web site and the paper guide, then certain students might have felt dissatisfied with receiving the less appealing task. The experiment took place over 3 days. One school class after the other

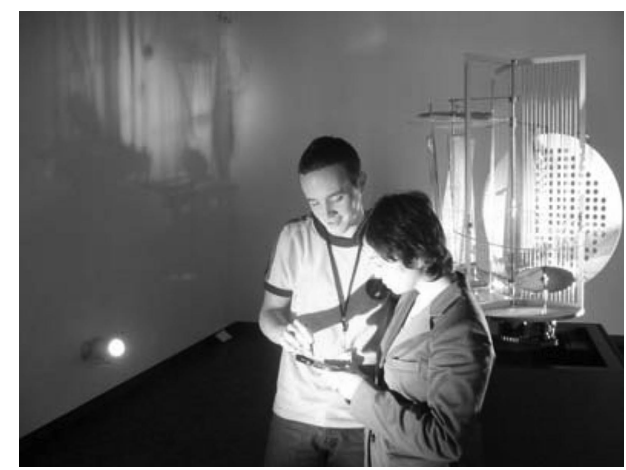

Figure 5. Interaction with the devices. 
visited the exhibition, which was closed to other visitors during those periods.

After entering the museum, students filled in a short demographic questionnaire in which they were asked if they had actually used the previsit planning Web site. Students who had used the Web site were randomly assigned to the preparationSelf conditions selfReco and selfRecoProScribe. Students who had previously received the paper guide were automatically assigned to the selfRecoPaper condition. As a result of technical problems with the PDAs, some other students were also assigned to the selfRecoPaper condition. Those students were asked to quickly recapture their preferred art objects and mark them in the paper guide before entering the exhibition. Students who did not use the Web site were randomly assigned to the noReco, ubiNextReco, and curatorReco conditions.

One student in every couple was given the handheld device; the other one received the audio recorder. In the selfRecoPaper condition, the couple had to agree to use the tour of one student. Afterwards, they were given a short introduction to using the ubiNext and had the opportunity to ask questions. They then entered the exhibition and could remain there as long as they wanted. After retrieving information about a certain art object, the students could take a quiz containing different types of questions, such as multiple choice and true or false.

If the students encountered problems with the system, they could approach an experimenter for help. In seven instances, the system crashed completely and had to be restarted. The data of these participants were excluded from the analysis. When the students were satisfied with the exhibition, they could return the device and leave. They were thanked for their participation.

\section{Results}

Besides the measurements mentioned above, some summaries were calculated. AllArt is the sum of art and groupArt, allOther is the sum of all other speech measurements, and groupTotal is the sum of all group measurements.

A 4 (user control) $\times 3$ (gender) $\times 2$ (education level) betweenparticipant analysis of variance was conducted. Table 1 displays the different conditions for the user control factor. The conditions for the participant couple's gender were male, mixed, and female. The two education levels were low (Havo) and high (Gymnasium). Table 2 and Figure 6 present the means in the user control conditions. The education level had no significant influence on the measurements. User control had a significant influence on speechTotal, $F(3,30)=3.821, p=.020$; navigation, $F(3,30)=$ 5.227, $p=.005$; silence, $F(3,30)=2.982, p=.047$; and numberOfObjects, $F(3,30)=4.735, p=.008$.

Post hoc $t$ tests with adjusted Bonferroni alphas revealed that the mean for speechTotal was significantly higher for the curatorReco

Table 2

Means of the User Control Conditions

\begin{tabular}{|c|c|c|c|c|}
\hline Measurement & noReco & ubiNextReco & selfReco & curatorReco \\
\hline \multicolumn{5}{|c|}{ Conversation } \\
\hline \multicolumn{5}{|l|}{ Summaries } \\
\hline speechTotal & $1,436.97$ & $1,479.52$ & $1,235.56$ & $1,870.45$ \\
\hline allArt & 510.23 & 473.95 & 284.56 & 476.52 \\
\hline allOther & 926.72 & $1,003.12$ & 951.50 & $1,393.58$ \\
\hline groupTotal & 132.00 & 138.13 & 136.56 & 135.73 \\
\hline \multicolumn{5}{|l|}{ Group } \\
\hline groupart & 56.50 & 52.70 & 35.22 & 58.93 \\
\hline grouphandheld & 20.00 & 28.10 & 44.50 & 36.22 \\
\hline groupnavigation & 6.53 & 22.98 & 6.61 & 14.37 \\
\hline groupsilence & 0.00 & 0.00 & 0.00 & 0.00 \\
\hline groupnotAudible & 5.35 & 2.68 & 1.50 & 3.83 \\
\hline groupoffTopic & 43.13 & 26.53 & 48.78 & 30.02 \\
\hline grouptroubleshooting & 0.47 & 5.80 & 0.00 & 1.93 \\
\hline \multicolumn{5}{|l|}{ Couple } \\
\hline art & 453.73 & 421.25 & 249.33 & 417.58 \\
\hline handheld & 88.83 & 77.52 & 109.22 & 72.75 \\
\hline navigation & 34.33 & 64.12 & 67.78 & 94.80 \\
\hline silence & 520.92 & 533.02 & 528.22 & 893.18 \\
\hline notAudible & 74.52 & 35.98 & 19.89 & 69.17 \\
\hline offTopic & 85.17 & 164.40 & 75.44 & 103.85 \\
\hline troubleshooting & 47.47 & 41.98 & 49.56 & 73.47 \\
\hline \multicolumn{5}{|c|}{ Behavior } \\
\hline \multicolumn{5}{|l|}{ Objects } \\
\hline numberOfObjects & 12.55 & 14.88 & 15.06 & 25.58 \\
\hline averageDurationAtObject & 101.36 & 90.57 & 76.32 & 68.75 \\
\hline \multicolumn{5}{|l|}{ Quizzes } \\
\hline numberOfQuizzes & 7.78 & 2.22 & 2.89 & 6.60 \\
\hline percentageOfQuizzes & 0.60 & 0.28 & 0.25 & 0.27 \\
\hline numberOfSelectionQuizzes & 3.57 & 1.42 & 0.89 & 2.02 \\
\hline \multicolumn{5}{|l|}{ Recommendations } \\
\hline numberOfRecommendation & - & 14.37 & 6.83 & 17.72 \\
\hline numberOfNoRecommendation & - & 0.52 & 8.22 & 7.87 \\
\hline numberOfFollowedRecommedation & - & 5.48 & 6.44 & 17.10 \\
\hline percentageOfFollowedRecomendation & - & 0.35 & 0.92 & 0.93 \\
\hline
\end{tabular}




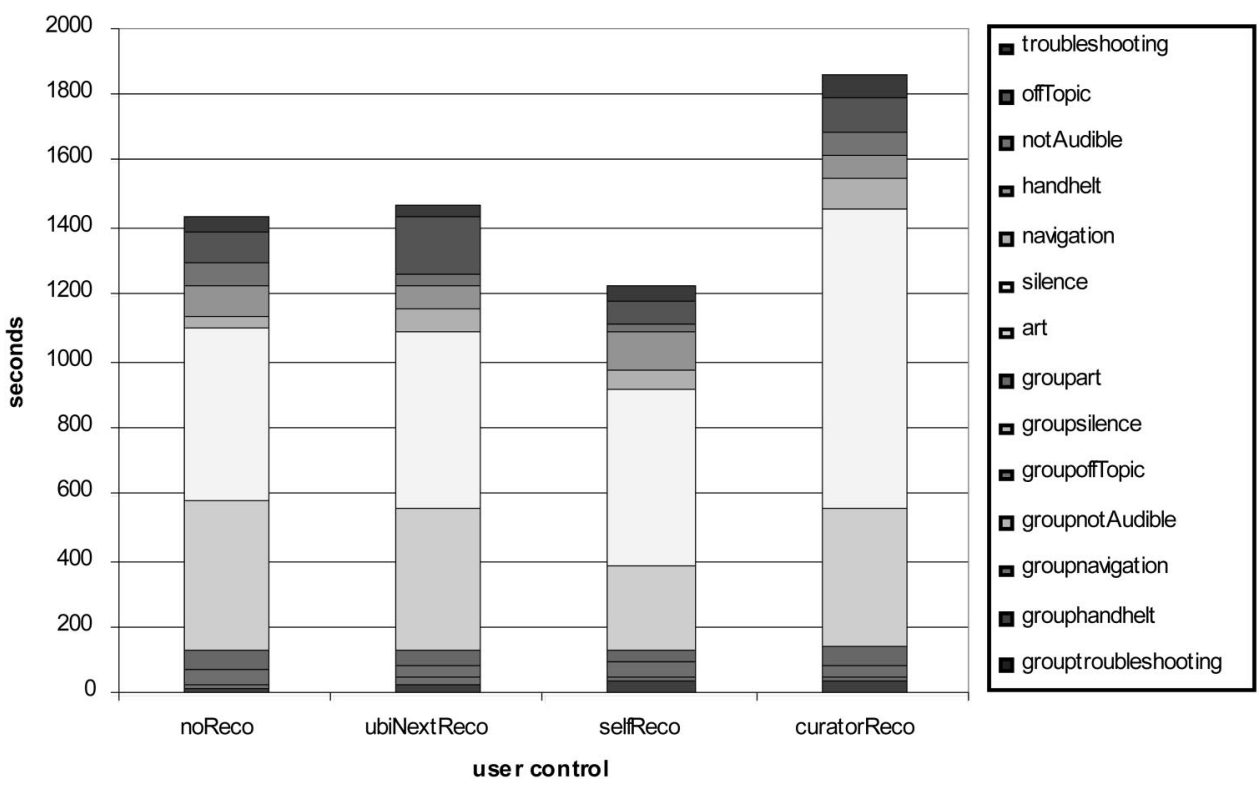

Figure 6. Cumulative means of the user control conditions.

condition as compared with the selfReco condition $(p=.015)$. The other two comparisons were nearly significant (ubiNextReco $p=.058$; noReco $p=.94$ ). The mean for navigation was significantly higher in the noReco condition as compared with the curratorReco condition ( $p=.006$ ). The mean for silence was significantly higher in the curatorReco condition as compared with the noReco $(p=.025)$, ubiNextReco $(p=.041)$, and selfReco ( $p=.049)$ conditions. The mean for numberOfObjects was significantly higher in the curatorReco condition as compared with the noReco $(p=.002)$, ubiNextReco $(p=.004)$, and selfReco $(p=.005)$ conditions.

Gender only had significant influence on navigation, $F(2,30)=$ $5.004, p=.013$, but failed to achieve significance in the post hoc $t$ test (see Table 3 ).

A 3 (technology) $\times 3$ (gender) $\times 2$ (education level) betweenparticipant analysis of variance was conducted. Naturally, the selfRecoPaper condition did not result in any entries in the server's

Table 3

Means in the Gender Conditions for the Speech Measurements

\begin{tabular}{lrrr}
\hline \multicolumn{1}{c}{ Measurement } & Male & Mixed & Female \\
\hline groupart & 42.85 & 83.33 & 50.50 \\
grouphandhelt & 38.81 & 26.00 & 23.80 \\
groupnavigation & 6.85 & 16.00 & 19.49 \\
groupnotAudible & 4.45 & 5.00 & 1.89 \\
groupoffTopic & 51.17 & 7.33 & 30.49 \\
groupsilence & 0.00 & 0.00 & 0.00 \\
grouptroubleshooting & 1.21 & 0.00 & 4.48 \\
art & 351.58 & 606.33 & 368.45 \\
handhelt & 86.48 & 94.67 & 78.19 \\
navigation & 51.73 & 106.00 & 62.54 \\
notAudible & 62.55 & 47.67 & 44.94 \\
offTopic & 147.10 & 104.67 & 71.80 \\
silence & 619.53 & 781.33 & 574.29 \\
troubleshooting & 52.83 & 37.00 & 61.37 \\
\hline
\end{tabular}

$\log$ file; therefore, the analysis needs to be limited to the speech data. Figure 7 shows the means for the technology conditions.

Education level had a significant influence on silence, $F(1$, $18)=9.075, p=.007$. The score for "lower" level (304) was below that for "higher" level (512). The students in the higher education level were more silent. Gender had no significant influence.

Technology had a significant influence on speechTotal, $F(1$, $18)=4.865, p=.020 ;$ handheld, $F(1,18)=5.427, p=.014$; navigation, $F(1,18)=4.409, p=.028$; and silence, $F(1,18)=$ $5.820, p=.011$. Post hoc $t$ tests with adjusted Bonferroni alphas revealed that speechTotal, handheld, and silence were significantly lower for selfRecoPaper as compared with selfReco $(p s=.010$, .000 , and .017 , respectively) and selfRecoProScribe ( $p$ s $=.035$, .002 , and .022 , respectively). Navigation was significantly lower for selfRecoPaper as compared with selfRecoProScribe $(p=.010)$.

An independent sample $t$ test was conducted with preparation as the independent variable. Table 4 shows the means and the result of the $t$ test. Figure 8 visualizes the means.

\section{Discussion and Conclusions}

The affordable MP3 player we used to record participants' conversations produced sufficient audio quality. The notAudible measurement is rather low. The most disturbing noises were the clicks when the device knocked against necklaces, chains, and zippers. We recommend asking participants to wear their necklaces and chains below their shirts and the audio device on top.

When asked to form couples, the students had a strong preference for the same gender. Only four mixed-gender couples emerged. However, these four couples talked much more about art than the other couples. Because of the small number of mixed couples, this difference failed to reach significance level; given a higher number of mixed couples, we would expect it to reach that level. Our preliminary results suggest that mixing genders within the couples would focus the conversations on art. 


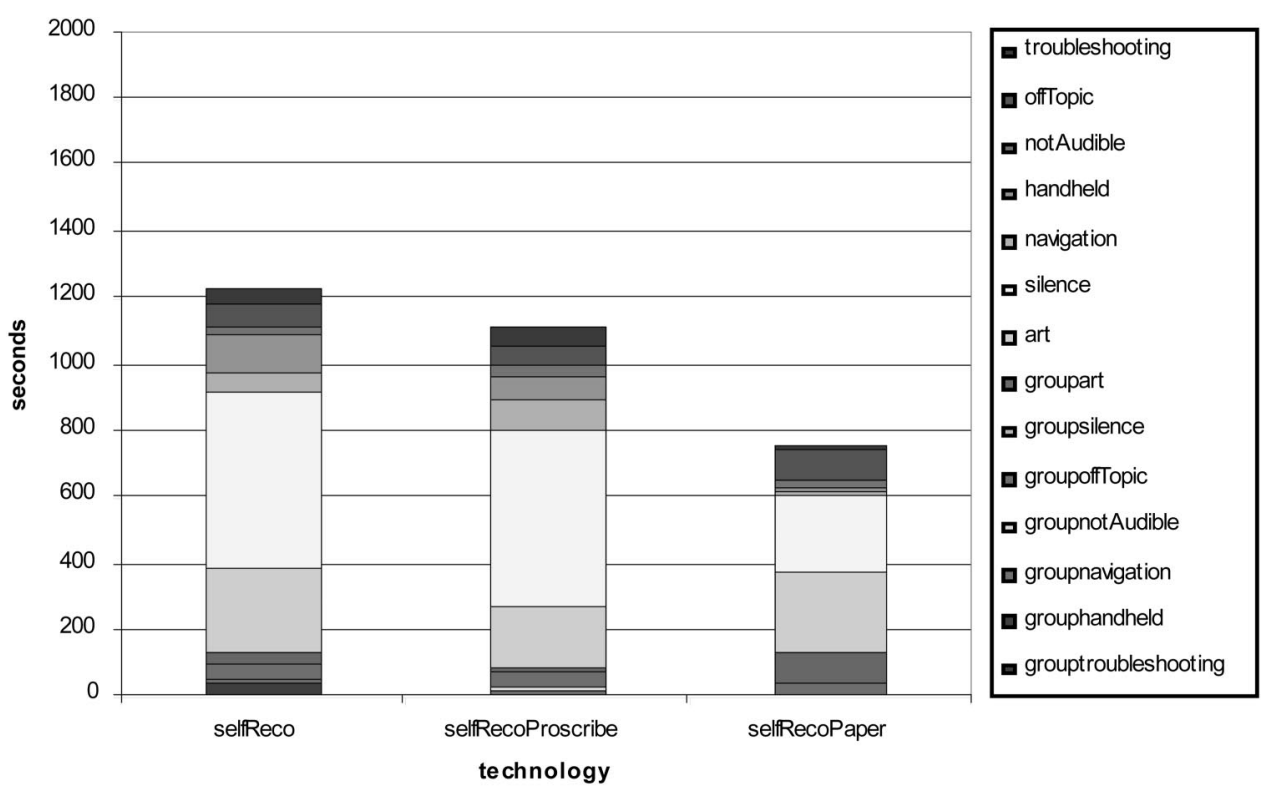

Figure 7. Cumulative means of the measurements in the technology conditions.

Students in this study were from roughly the same age group. This generation grew up with laptops, Game Boys, and mobile phones and is therefore familiar with handheld technology. Educating students is an important task for museums, but the needs of the general public are certainly more diverse. In particular, elderly visitors might have difficulties operating the system. The small font size and the unfamiliar pen-based interaction may challenge their sensoric and motoric abilities. In our next study, we plan to make ubiNext available to the general public and compare the results to the present study.

Table 4

Means and $t$ and $p$ Values in the Preparation Conditions

\begin{tabular}{lrrrr}
\hline \multicolumn{1}{c}{ Measurement } & Other & Self & $t$ & $p$ \\
\hline Summaries & & & & \\
speechTotal & $1,553.92$ & $1,002.72$ & 4.298 & 0.000 \\
allArt & 462.89 & 289.45 & 3.358 & 0.001 \\
allOther & $1,089.82$ & 713.24 & 3.505 & 0.001 \\
grouptotal & 138.92 & 115.62 & 1.070 & 0.289 \\
Group & & & & \\
grouptroubleshooting & 3.63 & 1.59 & 0.904 & 0.369 \\
grouphandheld & 29.61 & 17.28 & 1.571 & 0.121 \\
groupnavigation & 15.58 & 8.93 & 1.535 & 0.130 \\
groupnotAudible & 3.68 & 2.90 & 0.508 & 0.613 \\
groupoffTopic & 37.08 & 40.14 & -0.310 & 0.757 \\
groupsilence & 0.00 & 0.00 & - & - \\
groupart & 53.21 & 46.86 & 0.442 & 0.660 \\
Couple & & & & \\
art & 409.68 & 242.59 & 3.638 & 0.001 \\
handheld & 83.39 & 62.24 & 1.304 & 0.197 \\
navigation & 58.79 & 60.38 & -0.124 & 0.902 \\
silence & 628.76 & 394.14 & 2.962 & 0.004 \\
notAudible & 57.03 & 25.00 & 1.979 & 0.052 \\
offTopic & 114.74 & 63.93 & 1.572 & 0.121 \\
troubleshooting & 57.53 & 36.72 & 1.570 & 0.121 \\
\hline
\end{tabular}

The level of user control did not significantly influence the duration of talking about art. Still, participants remained significantly longer in the exhibition in the curatorReco condition. During that time, the participants looked at more art objects (numberOfObjects) and remained significantly longer in silence. The participants appeared to consume the information in a more passive mode.

Giving the user complete control on what object to look at (noReco) made it unnecessary to search for the recommended art objects. This resulted in significantly less time spent discussing navigation. Although they talked the same amount about art, the participants spent less time on navigation in the noReco condition and saw more objects in the curatorReco condition as compared with the ubiNextReco condition. The added value of the ubiNext recommendation could therefore not be detected in this study. The recommendation algorithm might have had insufficient feedback to calculate good recommendations. The participants viewed and rated barely more than 20 objects. If the system were able to receive more implicit (user observation) and explicit (object ratings) feedback, it might perform better. This would only be possible if the system's scope were expanded. This study was confined to only one exhibition within the museum. UbiNext's recommendation system might perform differently if more objects in several exhibitions became available. The physical distance between the art objects would become a real challenge. In such a setting, the recommendations should prevent sending the users from one end of the museum to the other while still providing good recommendations. We would like to test ubiNext in a larger part of the museum in our next study.

There was no significant difference in the measurements between the selfReco and selfRecoProScribe conditions. This indicates that the larger screen did not improve the learning experience. We were not able to detect an advantage of the ProScribe that would justify its three-times-higher price. However, this study 


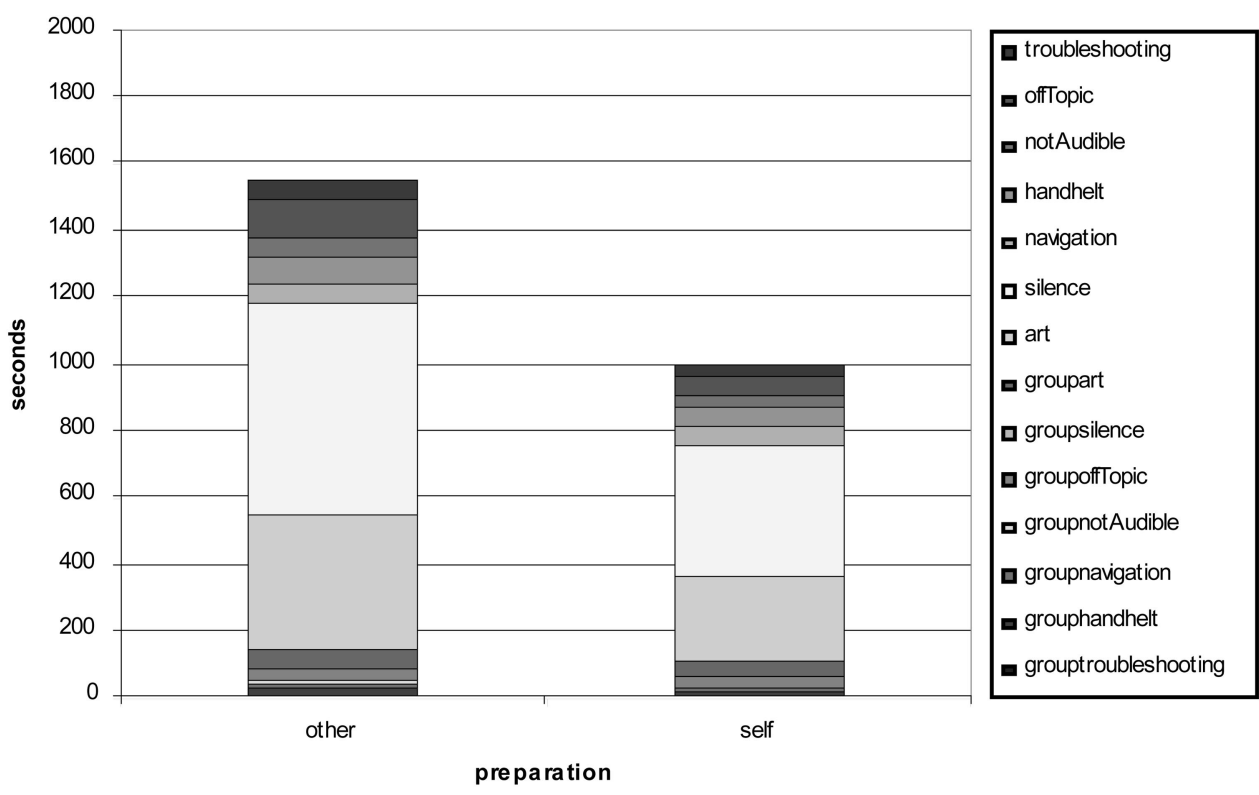

Figure 8. Cumulative means of the preparation conditions.

only ran for 3 days. Maintenance costs and other factors that are taken into account in the total cost of ownership could not be recorded. Maybe the ProScribe's robustness would in the end pay off, but a long-term financial study would be necessary to find an answer for this issue.

Using a paper-based guide resulted in the same amount of talking about art while drastically reducing handheld, navigation, and silence. The electronic devices failed to clearly show their benefit in this study. However, only static visual data were presented to the users. This forced the users to divide their attention between the art objects and the PDA. The use of additional audio commentary and movies might make the system more appealing and facilitate a better learning experience. Classic paper guides are naturally not able to present multimedia. In our next study, we intend to use the PDAs to their full potential by including multimedia content.

The participants who prepared themselves by using ubiNext's previsit planning Web site talked less about art as compared with the participants in the other conditions. This contradicts what Factor 3 of our framework predicted. However, one has to consider that the preparation was not voluntary. Students have a natural tendency to keep homework to the bare minimum. The tour that the students created for themselves was therefore smaller as compared with the curatorReco condition. When including a museum visit in art classes, it might be more useful to use noReco or curatorReco. The situation for volunteer visitors might be quite different. If users had a personal interest in the exhibition and museum and had the opportunity to prepare themselves for it, they might have a better learning experience. In our next study, we want to make the ubiNext previsit tour-planning Web site available to the general public and compare the results to the data gathered in this study.

\section{References}

Falk, J. H., \& Dierking, L. D. (2000). Learning from museums: Visitor experiences and the making of meaning. Walnut Creek, CA: AltaMira Press.

Griffin, J. (1996). School-Museum Informal Learning Experiences: A Teachers' Guide to Planning School Visits to Museums. University of Technology, Sydney.

Griffin, J. (1998). School-museum integrated learning experiences in science: A learning journey. Unpublished doctoral dissertation, University of Technology, Sydney, New South Wales, Australia.

Jensen, N. (1994). Children's perceptions of their museum experiences: A contextual perspective. Children's Environments, 11, 300-324.

Masuoka, A., Fukaya, T., Takahashi, T., Takahashi, M., \& Ito, S. (2005, July). Ubinext: A new approach to support visitor's learning experience in museums. Paper presented at the 11th International Conference on Human-Computer Interaction, Las Vegas, NV.

Roschelle, J. (1995). Learning in interactive environments: Prior knowledge and new experience. In F. J. H. \& L. D. Dierking (Eds.), Public institutions for personal learning (pp. 37-51). Washington, DC: American Association of Museums.

Smith, J. K., \& Smith, L. F. (2003). Origins of impressionism: Relating visitor behavior to perceived learning. Bulletin of Psychology and the Arts, 4(2), 80-85.

Received April 28, 2006

Revision received April 28, 2006

Accepted April 28, 2006 